\title{
Performance Analysis of Variable-Angle Quadrature Amplitude Constellations
}

\author{
Koralia N. Pappi ${ }^{1}$, Athanasios S. Lioumpas ${ }^{1}$, George K. Karagiannidis ${ }^{1}$ and Stavros A. Kotsopoulos ${ }^{2}$ \\ ${ }^{1}$ Department of Electrical \& Computer Engineering, Aristotle University of Thessaloniki, Greece. \\ \{kpappi;alioumpa;geokarag@auth.gr\} \\ ${ }^{2}$ Department of Electrical \& Computer Engineering, University of Patras, Rion, 26500 Patras, Greece.
}

\{Kotsop@ee.upatras.gr\}.

\begin{abstract}
We study the performance of a parametric quadrature amplitude modulation (QAM) family, called $\theta$-QAM, which includes other known constellations, such as square QAM (SQAM) and triangular QAM (TQAM), as special cases. The versatile structure of the $\theta$-QAM signal constellation, which occurs from the varying angle between the signal points, results in achieving the minimum symbol error rate (SER) or bit error rate (BER), under an average power constraint. Exact analytical expressions are obtained for the SER performance of $\theta$-QAM in additive white Gaussian Noise (AWGN) and Nakagami- $m$ fading channels, while highly accurate approximations for the BER are presented. The theoretical study aims at providing insight into the tradeoff between error performance and complexity of this parametric modulation scheme. The presented theoretical analysis is validated via extensive computer simulations.
\end{abstract}

\section{INTRODUCTION}

The utilization of higher-order modulation formats is one of the most common methods for increasing the bit rate in communications systems without increasing the required bandwidth. In the early 1960's, particular attention had been turned to the family of suppressed carrier two-dimensional signal constellations, such as the combined amplitude and phase modulation (AM-PM) [1]- [3] and the quadrature amplitude modulation (QAM) [4], [5]. Although a large variety of QAM constellations can be constructed, some specific constellations attracted special interest, mainly due to the low complexity demodulation methods required. One of the most widely used QAM constellation is the square-QAM (SQAM), with $4^{k}$ symbols, where $k$ is an integer. However, SQAM is not the optimum QAM constellation, in terms of transmission efficiency; that is, SQAM does not achieve the minimum error probability under an average power constraint [8].

Towards this concept, Foschini et al [8], by employing asymptotic approximations for the high signal-to-noise ratio (SNR) region, used a gradient-search procedure, which was initiated from several randomly chosen $N$-point arrays and converged in each case to a locally optimum constellation. Moreover, they rigorously proved that the optimum constellations formed a lattice of (almost) equilateral triangles, which offer a $0.5 \mathrm{~dB}$ (for 16 points) improvement in SNR over the conventional efficient QAM signaling formats, such as the (1-7)-QAM, the triangularQAM (TQAM) and the rectangular-QAM (RQAM), considering additive white Gaussian noise (AWGN) channels . Later, in [9], the authors studied via simulations the symbol error rate (SER) of various 16-ary and 64-ary QAM constellations, such as the circular-QAM (CQAM), the TQAM (also called hexagonal packing) and the RQAM, concluding that TQAM results in the best SER for average power-limited systems and they proposed a bit-to-symbol mapping through a heuristic technique. In [10], [11], the author studied the performance of TQAM, suggesting a detection method and a novel bit-to-symbol mapping. However, TQAM has an important disadvantage: the bit-to-symbol mapping cannot be optimal as in SQAM, resulting in a Gray code penalty, which deteriorates the bit error rate (BER). The performance of QAM schemes have been extensively studied also in numerous works (for example see [12]-[15]).

In this paper, we study a parametric QAM family, called $\theta$ QAM, which includes the SQAM and TQAM as special cases. For a specific value of the SNR, the maximum transmission efficiency is achieved by a different member of this family. The SER performance in AWGN is expressed by exact analytical formulas, and the BER by accurate approximations. Furthermore, we evaluate the SER and BER in Nakagami- $m$ fading, deriving exact closed-form expressions for the former and an approximation for the latter. The theoretical study aims at providing insight into the trade-off between complexity and performance over AWGN and fading channels, of this parametric modulation scheme, which includes the practical cases of TQAM and SQAM as special cases.

\section{THE $\theta$-QAM FAMILY}

Let us consider an $M$-ary SQAM constelaltion, where binary data are mapped into $M$ two-tuples $\left(x_{m}, y_{n}\right)$, each forming the transmitted symbol, $s_{m, n}$, and totally $M$ symbol vectors, $\mathbf{s}_{p}$, with $p=1, \ldots, M, M=4^{k}, k \in \mathbb{Z}^{+}$and $\{m, n\} \in$ $[1, \ldots, \sqrt{M}]$. The constellation structure is illustrated in Fig. 1 for $M=16$, where the SQAM symbols are depicted as the squared ones. The SQAM symbols are placed on the corners of squares with sides equal to $2 d$. Thus, the euclidean distance between any two adjacent symbols is $2 d$, with $d$ depending on the modulation order, $M$, and changes accordingly, so that the total average constellation's energy remains equal to $E_{a v}$. 
In $\theta$-QAM family constellations, the distances between symbols are as follows

$$
\begin{aligned}
& \mathcal{E}\left(s_{m, n}, s_{m, n+1}\right)=2 d, \quad n=1, \ldots, \sqrt{M}-1 \\
& \mathcal{E}\left(s_{m, n}, s_{m+1, n}\right)=2 d, \quad m=1, \ldots, \sqrt{M}-1
\end{aligned}
$$

but the symbols do not lie at the edges of a square but on the corners of isosceles triangles; the constellation's symmetry with respect to the point $(0,0)$ is maintained. The uneuqal angle, $\theta$, of those isosceles triangles affects the euclidean distance of the non-adjacent symbols as shown in Fig. 1, where $\theta$ QAM symbols are depicted for various values of the angle, $\theta$ (the TQAM constellation's symbols are shown as triangles). Practically, this angle affects the value of the total average energy per symbol, $E_{a v}$. For example, for the case of TQAM, which belongs to the $\theta$-QAM family, when $\theta=\pi / 3$, it is known that the average symbol energy is less than that of SQAM, finally leading in lower SER, since for the same $E_{a v}$, the minimum euclidean distance increases [10].

The coordinates of the symbols, $s_{m, n}$, of an 16-ary $\theta$-QAM constellation, can be derived after some simple trigonometric calculations as shown in Fig. 1 and can be generalized to the case of an $M$-ary $\theta$-QAM, since any higher order can be directly constructed by placing lower order constellatons adjacently. Finally, the coordinates $x_{m}, y_{m}$, of the symbol, $s_{m, n}$, of a $M$ ary $\theta$-QAM constellation are given by

$$
\begin{aligned}
\left(x_{m}, y_{n}\right)= & \left([2(n-1)+1-\sqrt{M}] d+[2 \bmod (m, 2)-1] \frac{a}{2},\right. \\
& \left.-[2(m-1)+1-\sqrt{M}] \frac{b}{2}\right),
\end{aligned}
$$

for $m=1, \ldots, \sqrt{M} / 2, \quad n=1, \ldots, \sqrt{M}$ and

$$
\begin{aligned}
\left(x_{m}, y_{n}\right)= & \left([2(n-1)+1-\sqrt{M}] d+[2 \bmod (m, 2)-1] \frac{a}{2},\right. \\
& \left.-[2(m-\sqrt{M} / 2-1)+1] \frac{b}{2}\right),
\end{aligned}
$$

for $m=\sqrt{M} / 2+1, \ldots, M$ and $n=1, \ldots, \sqrt{M}$. Moreover, $\bmod (\cdot)$ denotes the modulus after division, $2 d$ is the euclidean distance between the adjacent signal points, $a=2 d \cos \theta$ and $b=2 d \sin \theta$.Note, that from a theoretical point of view, the angle, $\theta$, may take values inside the interval $(0, \pi)$, but due to symmetry only the angles lower than $\pi / 2$ are considered.

\section{A. Average Energy per Symbol}

A random $\theta$-QAM constellation is shown in Fig. 2. The symbol energy is, $E_{S}=x_{m}^{2}+y_{n}^{2}$, which can be written as

$$
E_{a v}=\frac{2}{M} \sum_{i=0}^{\sqrt{M}-1} \sum_{j=0}^{\frac{\sqrt{M}}{2}-1}\left\{\left[(2 i+1-\sqrt{M}) d-\frac{a}{2}\right]^{2}+\left[(2 j+1) \frac{b}{2}\right]^{2}\right\}
$$

which after some manipulations is simplified to $E_{a v}=[3 M+$ $(4-M) \cos 2 \theta] d^{2} / 6$, which can be solved with respect to $d$, to get $d=\sqrt{6 E_{a v} /[3 M+(4-M) \cos 2 \theta]}$.

\section{B. Decision Boundaries and SER Evaluation in AWGN Chan- nels}

The decision boundaries of a random $\theta$-QAM are shown in Fig. 2 (red lines). These boundaries have been drawn in

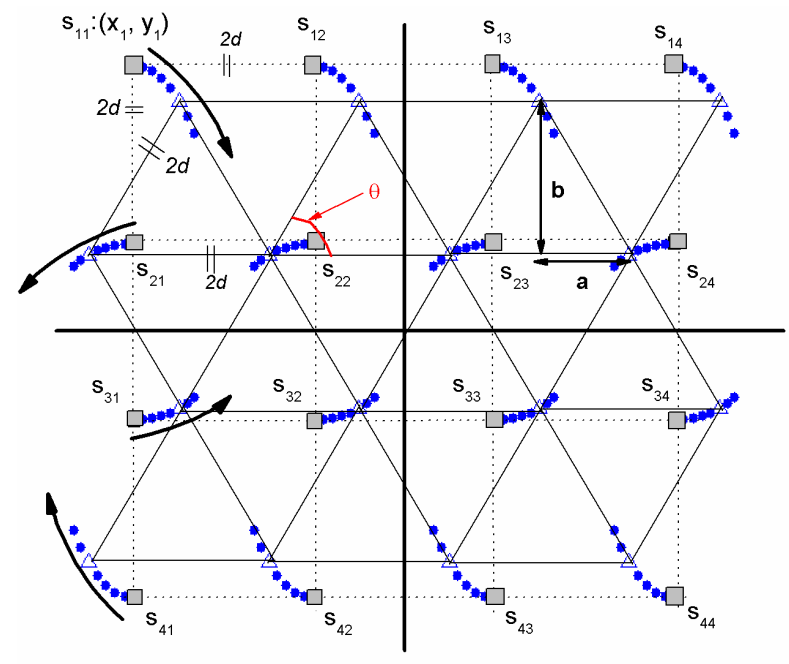

Fig. 1. The structure of the $\theta$-QAM constellation. SQAM symbols are depicted as squares, while the TQAM ones as triangles.

accordance with the maximum likelihood (ML) criterion, which minimizes the distance between the received signal and the demodulated symbol.

As it is shown, there are six different types of regions formed by the decision boundaries. These regions are denoted as $R_{1}$, $R_{2}, \ldots, R_{6}$ and they will be thus referred hereafter. It must be noted that the shapes of these regions are the same regardless of the modulation order; only the number of repetitions changes. For a general $M$-ary $\theta$-QAM constellation, it can be easily shown by observation, that the repetition number, $N_{R i}$ for the $i$-th region is given as in Table I.

The SER of this constellation can be evaluated by calculating for each transmitted symbol vector, $\mathbf{s}_{\mathbf{p}}$, the probability that the received vctor, $\mathbf{r}$, does not lie inside the corresponding decision region, $R_{p}$, and then taking the average of these probabilities, i.e.,

$$
P_{e}=\frac{1}{M} \sum_{p=1}^{M}\left(\int_{\frac{R_{p}}{}} f\left(\mathbf{r} \mid \mathbf{s}_{\mathbf{p}}\right) d r\right)
$$

where $f\left(\mathbf{r} \mid s_{p}\right)$ is the probability density function (pdf) of the received signal vector $\mathbf{r}$ conditioned on the transmitted signal, $\mathbf{s}_{\mathbf{p}}$, and $\overline{R_{p}}$ is the complementary of the decision region $R_{p}$. The pdf, $f\left(\mathbf{r} \mid \mathbf{s}_{\mathbf{p}}\right)$, is given by

$$
f\left(\mathbf{r} \mid \mathbf{s}_{\mathbf{p}}\right)=\frac{1}{\left(\pi N_{0}\right)^{N / 2}} e^{-\frac{1}{N_{0}}\left\|\mathbf{r}-\mathbf{s}_{\mathbf{p}}\right\|^{2}}=\frac{1}{\pi N_{0}} e^{-\frac{1}{N_{0}}\left(\left(x-x_{m}\right)^{2}+\left(y-y_{n}\right)^{2}\right)}
$$

where $N=2$ is the size of the vector $\mathbf{r}=(x, y)$ and $N_{0}$ is the power spectral density of the AWGN. Using cylindrical coordinates, the pdf in (3) can be written as

$$
f\left(\mathbf{r}(r, \phi) \mid s_{p}\right)=\frac{1}{\pi N_{0}} e^{-\frac{1}{N_{0}}\left(r-r_{S}\right)^{2}} .
$$

Below, the probabilities, $\frac{\int}{R_{p}} f\left(\mathbf{r} \mid \mathbf{s}_{\mathbf{p}}\right) d r$, in each type of region are calculated. Note, that it can be easily proved - by changing 


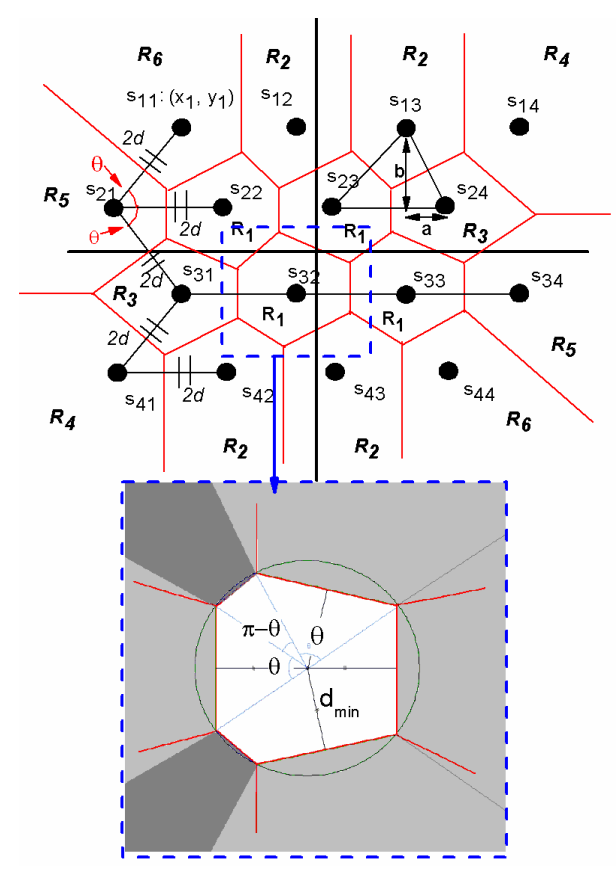

Fig. 2. The decision boundaries of a $\theta$-QAM constellation.

the variable in each integral- that regions of the same type have the same probability of error.

After dividing the $R_{1}$ into smaller parts, the total probability of error for this region is

$$
P_{R_{1}}=4 \int_{\frac{\pi-\theta}{2}}^{\frac{\pi+\theta}{2}} \int_{\frac{d}{\sin \phi}}^{\infty} g(r) r d r d \phi+2 \int_{\theta}^{\pi-\theta} \int_{\frac{d \sin \theta}{\sin \phi \cos \frac{\theta}{2}}}^{\infty} g(r) r d r d \phi
$$

where $g(r)=\frac{1}{\pi N_{0}} e^{-\frac{1}{N_{0}} r^{2}}$. Similarly, the probability of error for the other regions can be evaluated as in (6) (see the top of the next page). After some trivial mathematical manipulations, the SER can be written as in (7) (see next page), where $\gamma=$ $E_{a v} / N_{0}$ is the average SNR, $\delta=d / \sqrt{\operatorname{Eav}}, \csc (\cdot)=1 / \sin (\cdot)$ and

$$
\begin{aligned}
& c_{1}=1 /(2 \pi M), c_{2}=4(\sqrt{M}-2)(\sqrt{M}-1) \\
& c_{3}=\sqrt{2}(\sqrt{M}-1), c_{4}=5(\sqrt{M}-2)+6 \\
& c_{5}=3(\sqrt{M}-2)+2, \quad c_{6}=\sqrt{M} .
\end{aligned}
$$

It can be easily verified that for the case of $\theta=\pi / 2$, (7) reduces to known SER expressions of SQAM [17], while for $\theta=\pi / 3$ reduces to the SER of TQAM, which to the best of the authors' knowledge, has not been given in an analytical form in the past.

In Fig. 3, the SER is plotted against the angle, $\theta$, for specific SNRs. For low error probabilities, the effect of the angle, $\theta$, on the SER is obvious; for $M=16$ and $\mathrm{SNR}=20 \mathrm{~dB}$, the SER varies between $P_{e}=4 \times 10^{-6}$ and $P_{e}=10^{-3}$. On the other hand, for higher error probabilities, the effect of $\theta$ on the SER is negligible. In other words, there is no difference in the SER whether SQAM, or TQAM or any other $\theta$-QAM constellations is applied. If we take also into account the fact that in the high

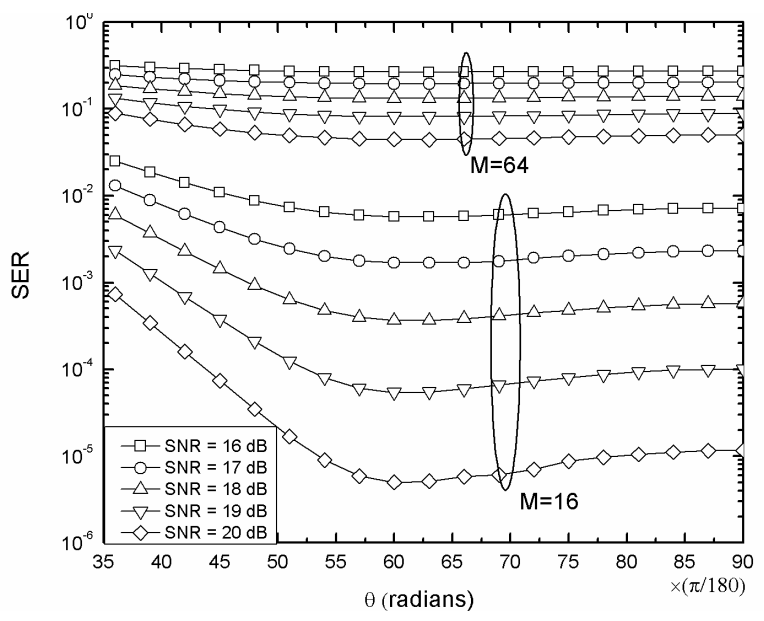

Fig. 3. The SER of $\theta$-QAM versus the angle, $\theta$ in AWGN.

SNR region, the optimum angle approaches $\theta=\pi / 3$, TQAM seem to be a sufficient compromise for achieving approximately the minimum SER over the whole SNR region. However, this claim is valid only if system's complexity is ignored (e.g. the demodulation of TQAM is more complex than that of SQAM).

TABLE I. THE NUMBER OF REPETITIONS OF THE $\theta$-QAM SIGNAL REGIONS

\begin{tabular}{|c|c|}
\hline Region & Number of repetitions \\
\hline 1 & $N_{R 1}=(\sqrt{M}-2)^{2}$ \\
\hline 2 & $N_{R 2}=2(\sqrt{M}-2)$ \\
\hline 3 & $N_{R 3}=\sqrt{M}-2$ \\
\hline 4 & $N_{R 4}=2$ \\
\hline 5 & $N_{R 5}=\sqrt{M}-2$ \\
\hline 6 & $N_{R 6}=2$ \\
\hline
\end{tabular}

\section{Bit StREAM MAPPING AND BER Evaluation OF $\theta$-QAM CONSTELLATIONS}

\section{A. Bit Stream Mapping}

In $\theta$-QAM constellations perfect Gray coding is not possible, since there are more than four adjacent neighbors around almost every signal point. A bit stream mapping for the special case of 16-TQAM has been proposed in [10]. This the optimum when $\theta>\pi / 3$, because the adjacent symbols located at a distance $2 d$, which is in that case the minimum distance between adjacent symbols, differ only in one bit . The adjacent symbols that differ in more than one bits are located at a distance different than $2 d$, minimizing the probability of having a symbol error of that kind. In general, the most probable symbol errors between adjacent symbols in $\theta$-QAM constellations, are those that occur between symbols that are located at a distance $2 d$.

\section{B. Gray Code Penalty}

Since perfect Gray coding is not possible for $\theta$-QAM, a Gray Code Penalty (GCP) will occur. Given the GCP, $G_{p}$, and the 


$$
\begin{aligned}
P_{R_{2}} & =\int_{\frac{\pi-\theta}{2}}^{\frac{\pi+\theta}{2}} \int_{\frac{d}{\sin \phi}}^{\infty} g(r) r d r d \phi+\int_{\theta}^{\pi-\theta} \int_{\frac{d \sin \theta}{\sin \phi \cos \frac{\theta}{2}}}^{\infty} g(r) r d r d \phi+2 \int_{\frac{\pi-\theta}{2}}^{\pi} \int_{\frac{d}{\sin \phi}}^{\infty} g(r) r d r d \phi \\
P_{R_{3}} & =\int_{\frac{\pi-\theta}{2}}^{\frac{\pi+\theta}{2}} \int_{\frac{d}{\sin \phi}}^{\infty} g(r) r d r d \phi+2 \int_{\theta}^{\pi-\theta} \int_{\frac{d \sin \theta}{\sin \phi \cos \frac{\theta}{2}}}^{\infty} g(r) r d r d \phi+2 \int_{\frac{\pi-\theta}{2}}^{\frac{\pi+2 \theta}{2}} \int_{\frac{d}{\sin \phi}}^{\infty} g(r) r d r d \phi \\
P_{R_{4}} & =\int_{\frac{\pi-\theta}{2}}^{\pi} \int_{\frac{d}{\sin \phi}}^{\infty} g(r) r d r d \phi+\int_{\frac{\pi-\theta}{2}}^{\frac{\pi+2 \theta}{2}} \int_{\frac{d}{\sin \phi}}^{\infty} g(r) r d r d \phi+\int_{2 \theta}^{\pi} \int_{\frac{2 d \sin \theta}{\sin \phi}}^{\infty} g(r) r d r d \phi \\
P_{R_{5}}= & \int_{\frac{\pi-\theta}{2}}^{\frac{\pi+\theta}{2}} \int_{\frac{d}{\sin \phi}}^{\infty} g(r) r d r d \phi+\int_{\frac{\pi-\theta}{2}}^{\pi} \int_{\frac{d}{\sin \phi}}^{\infty} g(r) r d r d \phi+\int_{\frac{\pi-\theta}{2}}^{\frac{\pi+2 \theta}{2}} \int_{\frac{d}{\sin \phi}}^{\infty} g(r) r d r d \phi+\int_{2 \theta}^{\pi} \int_{\frac{2 d \sin \theta}{\sin \phi}}^{\infty} g(r) r d r d \phi \\
P_{R_{6}}= & \int_{\theta}^{\pi-\theta} \int_{\frac{d \sin \theta}{\sin \phi}}^{\infty} g(r) r d r d \phi+2 \int_{\frac{\pi-\theta}{2}}^{\pi} \int_{\frac{d}{\sin \phi}}^{\infty} g(r) r d r d \phi
\end{aligned}
$$

$$
\begin{aligned}
P_{S}(\gamma, \theta, M)= & \frac{1}{M}\left(N_{R_{1}} P_{R_{1}}+N_{R_{2}} P_{R_{2}}+N_{R_{3}} P_{R_{3}}+N_{R_{4}} P_{R_{4}}+N_{R_{5}} P_{R_{5}}+N_{R_{6}} P_{R_{6}}\right) \\
= & c_{1}\left\{c_{2} \int_{\frac{\pi-\theta}{2}}^{\frac{\pi+\theta}{2}} \exp \left(-\gamma \delta^{2} \csc ^{2} \phi\right) d \phi+c_{3}^{2} \int_{\theta}^{\pi-\theta} \exp \left(-\gamma \delta^{2} \csc ^{2} \phi \sec ^{2} \frac{\theta}{2} \sin ^{2} \theta\right) d \phi\right. \\
& +c_{4} \int_{\frac{\pi-\theta}{2}}^{\pi} \exp \left(-\gamma \delta^{2} \csc ^{2} \phi\right) d \phi+c_{5} \int_{\frac{\pi-\theta}{2}}^{\frac{\pi+2 \theta}{2}} \exp \left(-\gamma \delta^{2} \csc ^{2} \phi\right) d \phi \\
& \left.+c_{6} \int_{2 \theta}^{\pi} \exp \left(-4 \gamma \delta^{2} \csc ^{2} \phi \sin ^{2} \theta\right) d \phi\right\}
\end{aligned}
$$

SER, $P_{S}$, the BER can be evaluated as [11]

$$
P_{b}=\frac{G_{p}}{\log _{2} M} P_{S} .
$$

The GCP for SQAM and TQAM is [11]

$$
G_{p}=\frac{1}{M} \sum_{i=1}^{M} G_{p}^{S_{i}}=\frac{1}{M} \sum_{i=1}^{M} \frac{\sum_{j=1}^{M\left(S_{i}\right)} B_{d}\left(S_{i}, S_{j}\right)}{N\left(S_{i}\right)}
$$

where $M$ is the constellation size, $S_{i}$ denotes $i$ th symbol, $G_{p}^{S_{i}}$ is the Gray Code Penalty of the symbol $S_{i}, N\left(S_{i}\right)$ is the number of the nearest neighbors of $S_{i}$ and $B_{d}\left(S_{i}, S_{j}\right)$ is the number of bit difference between $S_{i}$ and the nearest neighboring symbol of $S_{i}, S_{j}$. This is possible because, in SQAM and TQAM, the distance between every two adjacent symbols is always equal to $2 d$ (see Fig. 1).

On the other hand, in a random $\theta$-QAM constellation, it is not possible to use (9) for the evaluation of the GCP, because the adjacent symbols which are at a distance $2 d$ and those at a distance different than $2 d$ do not contribute in the same way to GCP. This happens because a symbol error is most probable to occur in regions that are closer to the transmitted symbol and the closer the adjacent symbols are to the transmitted symbol, the more they must contribute to the calculation of the GCP. Next, we propose an alternative method, which can be efficiently used for approximating the BER of the random $\theta$-QAM scheme.

\section{BER Approximation}

When evaluating the SER in each region (see Figure 2), we formed integrals on areas, the limits of which are radial, referring to the circumcircle of the region. For instance, in $R_{1}$, we formed two integrals for two different types of areas of integration. For the BER evaluation at high SNR values, we can assume that the errors occur very close to the limits of each region. Thus, we can multiply - as an approximation - each of these integrals with the corresponding number of different bits between the adjacent regions. If we follow this approach with all types of regions, the BER approximation will be given by (7), but now

$$
\begin{aligned}
& c_{1}=1 /\left(2 \pi M \log _{2} M\right) \\
& c_{3}=2(\sqrt{M}-1) \\
& c_{6}=2 \sqrt{M} .
\end{aligned}
$$

In Fig.4, the BER of $\theta$-QAM is plotted using the proposed method. As it can be seen, the approximation is highly accurate even for the medium and low SNR regions.

The most interesting result, regarding the BER is the fact that TQAM does not always achieve a lower error probability than SQAM as in the case of the SER. On the contrary, in some cases SQAM achieves the minimum BER among all the $\theta$-QAM constellations. As previously discussed, the reason is that for SQAM perfect Gray coding is possible, while for all the other $\theta$-QAM constellations, it is not. It is evident that for the high SNR region (SNR $>15 \mathrm{~dB}$ ) the minimum BER corresponds to an angle $\theta \approx 65(\pi / 180)$, while for the medium and low SNR region ( $\mathrm{SNR}<15 \mathrm{~dB}$ ), the optimum constellation is the SQAM one. From a practical aspect, TQAM is not the most appropriate constellation in terms of BER. 


\section{Performance over NaKagami- $m$ FAding CHANNELS}

\section{A. SER Evaluation of $\theta-Q A M$}

In the case of Nakagami-m fading, the SNR, $\gamma$, of the received signal will be a gamma distributed random variable, i.e., [19]

$$
f_{\gamma}(\gamma)=\frac{m^{m} \gamma^{m-1}}{\bar{\gamma}^{m} \Gamma(m)} \exp \left(-\frac{m \gamma}{\bar{\gamma}}\right)
$$

where $\bar{\gamma}$ is the average SNR, $m$ is the Nakagami- $m$ fading parameter, which ranges from 0.5 to $\infty$ and $\Gamma(\cdot)$ denotes the complete Gamma function.

The SER evaluation of $\theta$-QAM in Nakagami- $m$ fading, with integer values of $m$ can be evaluated by averaging (7) over the (10), which reduces to (11) (see at the top of next page), after solving the inner integral and using [16, 3.351 (3)].

Now, the integrals involved in (11) have the following form

$$
I_{1}=\int_{l_{1}}^{l_{2}}\left(\frac{m}{m+A \csc ^{2} x}\right)^{m} d x
$$

which can be written as

$$
\begin{aligned}
I_{1} & =\int_{l_{1}}^{l_{2}}\left(\frac{m \sin ^{2} x}{m \sin ^{2} x+A}\right)^{m} d x \stackrel{y=2 x}{=} \\
& =\frac{1}{2} \int_{2 l_{1}}^{2 l_{2}}\left(1+\frac{\alpha}{\beta+\cos y}\right)^{m} d y
\end{aligned}
$$

with $\alpha=2 A / m$ and $\beta=-1-2 A / m$. By using the binomial expansion of the term $\left(1+\frac{\alpha}{\beta+\cos y}\right)^{m}$, and after exchanging the order of summation and integration, we get

$$
I_{1}=\frac{1}{2} \sum_{k=0}^{m}\left(\begin{array}{c}
m \\
k
\end{array}\right) \int_{2 l_{1}}^{2 l_{2}} \frac{\alpha^{k}}{(\beta+\cos y)^{k}} d y
$$

The integral in (14) can be evaluated in closed-form in terms of elementary functions using [16, 2.554 (3)].

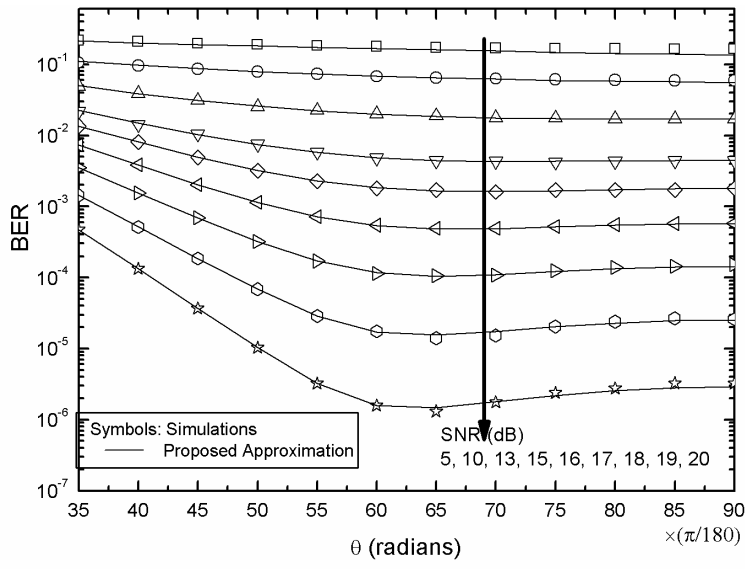

Fig. 4. The approximated BER of $\theta$-QAM for $M=16$ in AWGN.

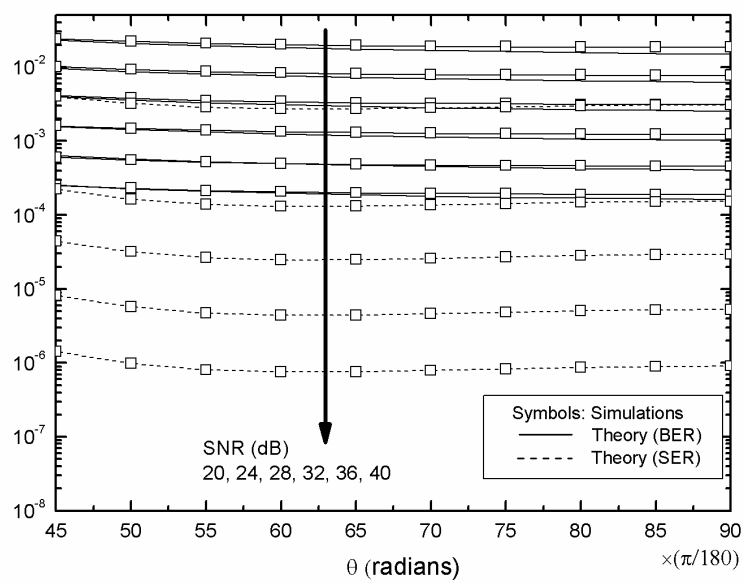

Fig. 5. The SER $(m=3)$ and BER $(m=1)$ of $\theta$-QAM for $M=16$ in Nakagami- $m$ fading.

1) Special case: Rayleigh fading $(m=1)$ : For the important case of Rayleigh fading, (14) reduces to [16, 2.554 (3)]

$$
\begin{aligned}
I_{1}= & \int_{l_{1}}^{l_{2}} \frac{1}{1+A \csc ^{2} x} d x=\left[l_{2}-\sqrt{\frac{A}{1+A}} \operatorname{atan}\left(\frac{\sqrt{1+A} \tan l_{2}}{\sqrt{A}}\right)\right]- \\
& {\left[l_{1}-\sqrt{\frac{A}{1+A}} \operatorname{atan}\left(\frac{\sqrt{1+A} \tan l_{1}}{\sqrt{A}}\right)\right], l_{1}, l_{2} \neq \frac{\pi}{2} }
\end{aligned}
$$

By applying (15) in (11) we get a closed-form solution for the SER of $\theta$-QAM in Rayleigh fading, in terms of elementary functions.

\section{B. BER Approximation}

Following the same procedure as that of the case of AWGN, the BER of $\theta$-QAM in Nakagami- $m$ fading can be approximated by (11) where now

$$
\begin{aligned}
& c_{1}=1 /\left(2 \pi M \log _{2} M\right) \\
& c_{3}=2(\sqrt{M}-1) \\
& c_{6}=2 \sqrt{M} .
\end{aligned}
$$

In Fig. 5, the SER and BER is depicted against the angle, $\theta$, assuming Rayleigh fading. It is interesting to note that the minimum BER is achieved by the SQAM. For example, when $\bar{\gamma}=40 \mathrm{~dB}$, TQAM results in a BER of $2 \times 10^{-4}$, while SQAM in a BER of $1.5 \times 10^{-4}$. In general, one can observe that for the case of fading channels the choice of any of the $\theta-Q A M$ constellations should be based on the demodulation complexity.

\section{Conclusions}

A parametric family of quadrature modulation was presented studied, called $\theta$-QAM, which includes other known quadrature modulations, such as SQAM and TQAM, as special cases. We presented exact analytical expressions for the SER and highly accurate approximation for the BER in AWGN and Nakagami- $m$ fading channels. The theoretical study aims at providing insight into the trade-off between error performance and complexity of this parametric modulation scheme. It was 


$$
\begin{gathered}
P_{S_{N}}=c_{1}\left\{c_{2} \int_{\frac{\pi-\theta}{2}}^{\frac{\pi+\theta}{2}}\left(\frac{m}{m+\delta^{2} \csc ^{2} \phi \bar{\gamma}}\right)^{m} d \phi+c_{3}^{2} \int_{\theta}^{\pi-\theta}\left(\frac{m}{m+\delta^{2} \csc ^{2} \sec ^{2} \frac{\theta}{2} \sin ^{2} \theta \phi \bar{\gamma}}\right)^{m} d \phi\right. \\
+c_{4}\left(\int_{\frac{\pi-\theta}{2}}^{\pi}\left(\frac{m}{m+\delta^{2} \csc ^{2} \phi \bar{\gamma}}\right)^{m} d \phi\right)+c_{5} \int_{\frac{\pi-\theta}{2}}^{\frac{\pi+2 \theta}{2}}\left(\frac{m}{m+\delta^{2} \csc ^{2} \phi \bar{\gamma}}\right)^{m} d \phi \\
\left.+c_{6}\left(\int_{2 \theta}^{\pi}\left(\frac{m}{m+4 \delta^{2} \csc ^{2} \phi \sin ^{2} \theta \bar{\gamma}}\right)^{m} d \phi\right)\right\}
\end{gathered}
$$

shown that, TQAM achieves approximately the minimum SER over the whole range of SNRs and that it is an efficient solution when the choice of the modulation is based on the SER minimization. On the other hand, the minimum BER is achieved for an angle different from $\theta=60^{\circ}$ (TQAM). Furthermore, it was shown that in fading channels, SQAM is the optimum constellation in terms of BER.

\section{REFERENCES}

[1] R. W. Lucky and J. C. Hancock, "On the optimum performance of N-ary systems having two degrees of freedom," IRE Trans. Commun. Syst., vol. CS-10, pp. 185-192, June 1962.

[2] J. Salz, J. R. Sheehan, and D. J. Paris, "Data transmission by combined AM and PM,” Bell Syst. Tech. J., vol. 50, Sept. 1971.

[3] K. Kawai, S. Shintani, and H. Yanagidaira, "Optimum combination of amplitude and phase modulation scheme," in 1972 Int. Conf: Communications Rec., Philadelphia, Pa.

[4] R. W. Lucky, J. Salz, and E. J. Weldon, Jr., Principles of Data Communication: New York: McGraw-Hill, 1968

[5] C. Campopiano and B. Glazer, "A coherent digital amplitude and phase modulation scheme," IRE Trans. Commun., vol. 10, pp. 90-95, Dec. 1962.

[6] A. J. Goldsmith and S.-G. Chua, "Variable-rate variable-power MQAM for fading channel," IEEE Trans. Commun., vol. 45, no. 10, pp. 1218-1230, Oct. 1997.

[7] Conti, A.; Win, M. Z.; Chiani, M.; "Slow Adaptive M -QAM With Diversity in Fast Fading and Shadowing," IEEE Trans. Commun., Volume 55, Issue 5, May 2007

[8] G. Foschini, R. Gitlin, and S. Weinstein, "Optimization of twodimensional signal constellations in the presence of gaussian noise," IEEE Trans. Commun., vol. 22, pp. 28-38, Jan. 1974.
[9] M. Kifle and M. Vanderaar, "Bounds and Simulation Results of 32-ary and 64-ary Quadrature Amplitude Modulation for Broadband-ISDN via Satellite," NASA Technical Memorandum 106484, February, 1994.

[10] Sung-Joon Park, "Triangular Quadrature Amplitude Modulation", IEEE Communications Letters, vol. 11, No4, April 2007.

[11] Sung-Joon Park, "Bit mapping of Triangular Quadrature Amplitude Modulation", The 18th Annual IEEE International Symposium on Personal, Indoor and Mobile Radio Communications (PIMRC'07), 1-4244-1144$0 / 07$.

[12] J. Lu, K. B. Letaief, J. C.-I. Chuang, and M. L. Liou, "M-PSK and $M$-QAM BER computation using signal-space concepts," IEEE Trans. Commun., vol. 47, no. 2, pp. 181-184, Feb. 1999.

[13] L.-L. Yang and L. Hanzo, "A recursive algorithm for the error probability evaluation of $M$-QAM," IEEE Commun. Lett., vol. 4, no. 10, pp. 304-306, Oct. 2000.

[14] K. Cho and D. Yoon, "On the general BER expression of one- and twodimensional amplitude modulations," IEEE Trans. Commun., vol. 50, no. 7, pp. 1074-1080, Jul. 2002.

[15] Conti, A.; Win, M.Z.; Chiani, M.; " Invertible bounds for M-QAM in Rayleigh fading,"IEEE Trans. on Wireless Commun., vol. 4, no. 5, Sept. 2005

[16] I. S. Gradshteyn and I. M. Ryzhik, Table of Integrals, Series, and Products, 6th ed. New York: Academic, 2000.

[17] J. G. Proakis, Digital Communications, 4th ed. New York: McGraw-Hill, 2001.

[18] J. A. Nelder and R. Mead, "A Simplex Method for Function Minimization," Computer Journal, vol. 7, pp. 308-313, 1965.

[19] M. K. Simon and M. Alouini, Digital Communication over Fading Channels, 2nd ed. New Jersey: John Wiley, 2005. 